\title{
An analogue future for 2D
}

Two-dimensional materials could first find widespread commercial application in analogue electronics, rather than as a replacement for silicon in digital devices.

T

he basic building block of the electronics industry - the field-effect transistor - is most closely associated with its role as a digital switch, where the current between source and drain is set to high or low, on or off, 1 or 0 . But the transistor can also play the role of an amplifier, raising the voltage or current of a weak electronic signal to a stronger one. This is an analogue operation (albeit one that is performed in both analogue and digital circuits), where the electric signal takes a continuously variable value.

Despite being overshadowed by its digital counterpart, analogue electronics is essential in many devices. Analogue switches are, for example, used in the front-end of mobile phones to amplify and route radio-frequency signals, before being converted to a digital signal ${ }^{1-3}$. And it is within analogue electronics - rather than as a replacement for silicon in digital circuits - that two-dimensional electronic materials could first find widespread commercial application.

The potential of 2D materials in analogue electronics is highlighted in this issue of Nature Electronics. To start, Deji Akinwande and colleagues at the University of Texas, Austin and the University of Lille report that monolayers of hexagonal boron nitride can be used to make analogue switches that operate at the frequencies used in $5 \mathrm{G}$ communication systems, as well as the communication systems beyond it. Owing to the atomic thinness of the boron nitride, the devices have a high cutoff frequency of $129 \mathrm{THz}$ (a figure-of-merit used to compare different radio-frequency switches), and a d.c. energy consumption per switching event of less than a nanojoule.

In an accompanying News \& Views article, Frank Schwierz at the Technische Universität Ilmenau notes that since the pioneering work on graphene in 2004,

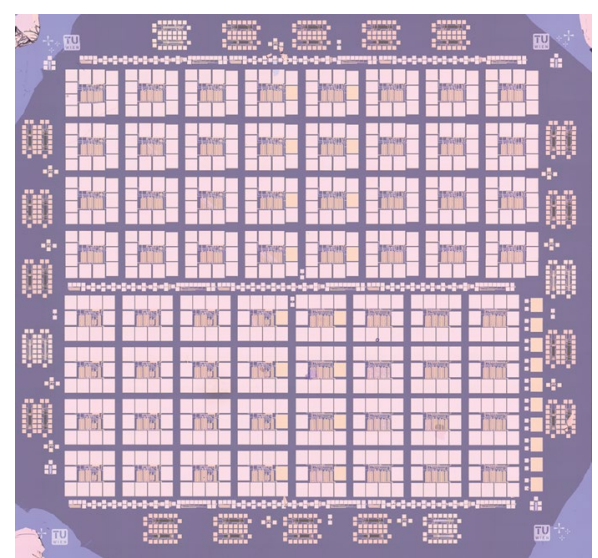

An optical microscopy image of a chip containing 64 operational amplifiers that are made using two-dimensional molybdenum disulfide as the active material ${ }^{6}$. Credit: Thomas Mueller

around 1,500 different 2D materials have been identified, and most research on their electronics properties has focused on trying to replace silicon as the channel material of transistors in digital electronics. But, as he explains: "If practical applications are the target, a more promising approach is to look for niche applications where traditional materials perform poorly."

Analogue switches are one such application, operational amplifiers could be another. In a second Article in this issue, Thomas Mueller and colleagues show that operational amplifiers can be made from 2D molybdenum disulfide $\left(\mathrm{MoS}_{2}\right)$ field-effect transistors. The researchers - who are based at the Vienna University of Technology, Università di Pisa and $\mathrm{AMO} \mathrm{GmbH}$ Aachen - use these operational amplifiers to build several analogue circuits, including inverting amplifiers, integrators, log amplifiers, and transimpedance amplifiers.
The gain and bandwidth of the 2D operational amplifiers do not currently match that of commercial silicon devices, but there are still areas where they could be beneficial. The physical world is, of course, an analogue one and sensors are usually measuring analogue signals that need to be preconditioned before digital conversion. This preconditioning is typically performed using rigid silicon electronic circuitry, which limits the capabilities of flexible sensors. Mueller and colleagues demonstrate that their approach can, instead, be used to create integrated circuits that combine a photodetector and signal preconditioning circuit on the same $\mathrm{MoS}_{2}$ film, which itself is placed on a flexible polyimide substrate.

Analogue electronics is also important in approaches to neuromorphic computing $^{4}$, and this is another area in which 2D materials could be a valuable alternative to silicon. Shi-Jun Liang, Feng Miao and colleagues have, for instance, recently shown that electrically tunable homojunction devices created from $2 \mathrm{D}$ tungsten diselenide ( $\mathrm{WSe}_{2}$ ) can be used to build circuits that exhibit neuromorphic capabilities $^{5}$. The researchers - who are based at Nanjing University and the National Institute for Materials Science, Japan - demonstrate, in particular, that three of these homojunction devices can create synaptic circuits that conventional silicon technology would require more than ten transistors to recreate.

Published online: 18 August 2020 https://doi.org/10.1038/s41928-020-00469-6

References

1. Bowick, C. RF circuit design (Elsevier, 2011).

2. Bourzac, K. Nature 483, S34-S36 (2012).

3. Kim, M. et al. Nat. Commun. 9, 2524 (2018).

4. Mead, C. Nat. Electron. 3, 434-435 (2020).

5. Pan, C. et al. Nat. Electron. 3, 383-390 (2020).

6. Polyushkin, D. K. et al. Nat. Electron. https://doi.org/10.1038/ s41928-020-0460-6 (2020). 